\title{
Implantable collamer lens versus small incision lenticule extraction for high myopia correction: A systematic review and meta- analysis
}

Kai Cao, Jingshang Zhang, Jinda Wang, Mayinuer Yusufu, Shanshan Jin, Shuying Chen, Ningli Wang, Zi-Bing Jin and Xiu Hua Wan*

\begin{abstract}
Purpose: To compare the efficacy, safety, predictability and visual quality between implantable collamer lens (ICL) implantation and small incision lenticule extraction (SMILE) for high myopia correction in adults.

Methods: A systematic review and meta-analysis was conducted. A comprehensive literature search was done based on databases including PubMed, Science Direct, Embase, and the Cochrane Central Register of Controlled Trials. The efficacy index, safety index, changes in Snellen lines of corrected distance visual acuity (CDVA), predictability (difference between post-operative and attempted spherical equivalent error, SER), incidence of halos, and change in higher-order aberrations (HOAs) were compared. Mean difference (MD) and 95\% confidence interval (CI) was used to estimate continuous outcomes, risk ratio (RR) and $95 \% \mathrm{Cl}$ was used to estimate categorical outcomes.

Results: Five observational studies involving 555 eyes were included in this review. Studies' sample sizes (eyes) ranged from 76 to 197. Subjects' refraction ranged from -6 diopter (D) to -12D. Study duration of most researches were 6 months or 12 months. Compared to SMILE, ICL implantation showed better efficacy index (MD=0.09, 95\%Cl:0.01 to 0.16 ) and better safety index ( $M D=0.08,95 \% \mathrm{Cl}: 0.00$ to 0.16$)$. Compared with $S M I L E$, more ICL-treated eyes gained one or more Snellen lines of CDVA ( $R R=1.54,95 \% \mathrm{Cl}: 1.28$ to 1.86), more gained two or more lines ( $R R=2.09,95 \% \mathrm{Cl}: 1.40$ to 3.13), less lost one or more lines ( $R R=0.17,95 \% \mathrm{Cl}: 0.05$ to 0.63$)$. There was no difference in predictability between two treatments, RRs of predictability of within $\pm 0.5 \mathrm{D}$ and $\pm 1 \mathrm{D}$ were 1.13 ( $95 \% \mathrm{Cl}: 0.94$ to 1.36 ) and 1.00 (95\% Cl: 0.98 to 1.02). Compared with SMILE, ICL implantation came with a higher risk of halos [RR=1.79, 95\%Cl: 1.48 to 2.16$]$ and less increase in total $\mathrm{HOAs}(\mathrm{MD}=-0.23,95 \% \mathrm{Cl}:-0.42$ to -0.03$)$.
\end{abstract}

Conclusion: Compared with SMILE, ICL implantation showed a higher risk of halos, but equal performance on SER control, and better performance on efficacy index, safety index, CDVA improvement and HOAs control. Overall, ICL implantation might be a better choice for high myopia correction in adults.

Keywords: Intraocular lens, Implantable collamer lens, small incision lenticule extraction, high myopia

*Correspondence: wanxh@ccmu.edu.cn

Beijing Institute of Ophthalmology, Beijing Tongren Hospital, Beijing Key Laboratory of Ophthalmology and Visual Sciences, Capital Medical University, No17, Hougou ally, Dongcheng district, Beijing 100005, China

\section{Introduction}

Nowadays, it is well accepted to use small incision lenticule extraction (SMILE) to correct low-to-moderate myopia [1, 2]. For high myopia correction, SMILE also brought satisfying prognosis [3, 4], however, SMILE has 
its inherent limitations such as thick ablation depths, limited ablation zones and increased aberrations, SMILE could increase the risk of dry eye [5], myopic regression [6], haze [7] and corneal ectasia [8].

Implantable collamer lens (ICL) implantation was another promising way for high myopia correction [9], the postoperative spherical equivalent error (SER) was reported to be predictable $[10,11]$ and stable $[12,13]$. ICL implantation broadened the scope of target population, such as patients with thin cornea [14] and even keratoconus [15]. A few studies compared ICL implantation with SMILE for high myopia correction, conclusions from different studies were controversial. Siedlecki J [16] and coworkers reported ICL implantation yielded better uncorrected distance visual acuity, better refractive accuracy, and fewer higher-order aberrations (HOAs) than SMILE. Moshirfar M [17] and coworkers thought SMILE might be comparable to ICL for high myopia correction, similarly, Wei R [18] reported both treatments showed compared performance for high myopia correction.

In this review, we aim to make a strengthened comparison between ICL implantation and SMILE for high myopia correction in adults. The efficacy index, safety index, changes in Snellen lines of corrected distance visual acuity (CDVA), predictability, incidence of halos, and change in higher-order aberrations (HOAs) would be compared between two treatments.

\section{Methods and Materials}

\section{Inclusion criteria}

Studies were included under the following consideration: 1) Subjects with high myopia (SER should be equal or greater than -6 diopter, D); 2) Subjects' age $\geq 18$ years old; 3) The intervention measures must include ICL implantation and SMILE.

\section{Exclusion criteria}

Studies were excluded in any of the following condition: 1) Studies of case report, letter, comment or review; 2) Studies used only ICL implantation or only SMILE; 3 ) Studies that included low to moderate myopic patients (SER of -0.5D to -6D) or other kinds of patients.

\section{Databases and Search strategy}

We searched PubMed, Science Direct, and the Cochrane Central Register of Controlled Trials (from inception to 15 August, 2021) for studies published in English, the detailed search strategies were shown in the end of the manuscript.

\section{Outcomes}

According to the scale of extracted data, the following outcomes were quantitatively assessed: The efficacy index, safety index, changes in Snellen lines of CDVA, predictability, incidence of halos, and change in HOAs. Besides, the following outcomes were qualitatively described: endothelial cell loss, complications including cataract and dry eye, visual quality including objective scatter index (OSI) and modulation transfer function cutoff frequency $\left(\mathrm{MTF}_{\text {cut-off }}\right)$ value.

\section{Data Extraction}

The following information measured at last-follow up time of each study was extracted: First author, publication year, subjects' mean/median age, sample size (number of eyes), study design, mean follow-up duration, subjects' SER range and type of ICL. We extracted the data of outcomes for analysis using a pre-designed data form. Briefly, for categorical data, such as the number of halos, the number of events were extracted. For continuous data, such as the efficacy index, we extracted the mean value and standard deviation (SD).

\section{Data synthesis and statistical analysis}

Meta-analysis was performed using either a fixed-effects model or a random-effects model according to the heterogeneity across included studies. The heterogeneity was assessed by a Q-test and the $\mathrm{I}^{2}$ statistic. The $\mathrm{I}^{2}$ statistic describes the percentage of variability caused by heterogeneity rather than by chance. An $\mathrm{I}^{2}$ of $\leq 50 \%$ indicates a relatively small heterogeneity across studies, subsequently a fixed-effects model would be used, otherwise a random-effects model would be used [19]. We used the mean differences (MDs) and 95\% confidence intervals (CIs) to make comparison of continuous outcomes between ICL implantation and SMILE, we used risk ratios (RRs) and 95\% CIs to estimate categorical outcomes. We used Egger's test to determine publication bias. The significance level was set to be 0.05 , two-tailed. All statistical analysis was done using the open-source $\mathrm{R}$ program (Version 4.0.0).

\section{Results \\ Paper selection}

The paper selection process was shown in Figure 1. Initially, a total of 293 articles were identified from PubMed, Science direct and Cochrane Central Register of Controlled Trials. 62 duplicates were removed, then 181 publications were further excluded by title and abstract. 23 reviews, letters or case reports were excluded. 22 articles were further excluded due to the following reasons: study in Czech $(n=1)$, studies where only ICL implantation was performed $(n=2)$, studies where subjects were children $(n=2)$, studies recruited subjects of low or moderate myopia, or emmetropia $(n=16)$, studies of iris-fixated 


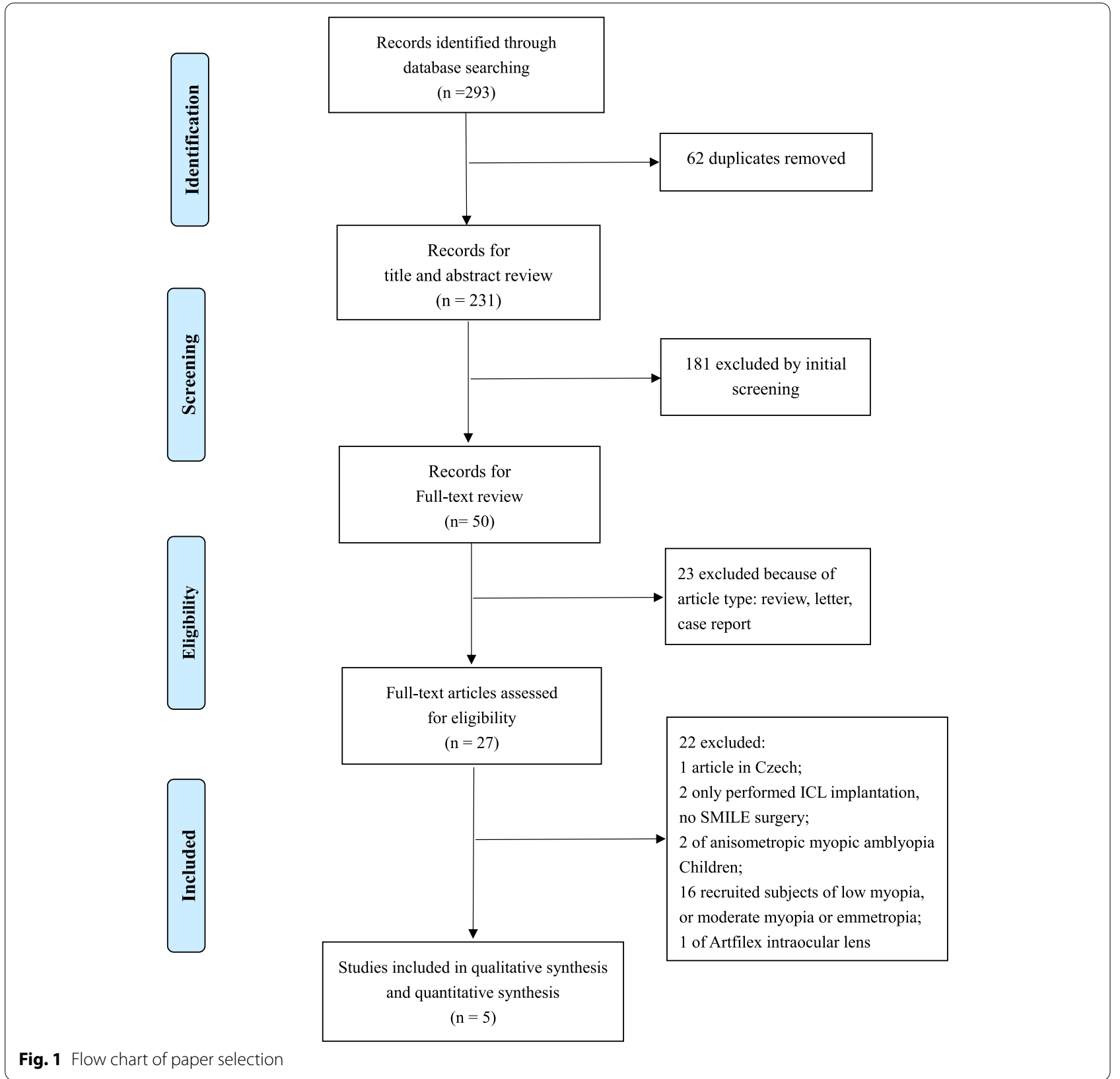

Artiflex lens $(\mathrm{n}=1)$. Finally, 5 studies $[13,16,18,20,21]$ (involving 555 eyes) were included in this meta-analysis.

\section{Characteristics of the included studies}

The characteristics of the five included studies were shown in Table 1. All studies were observational studies, and were published between year 2019 to 2021. All subjects were high myopic adults (age $\geq 18$ ), the sample sizes (number of eyes) ranged from 76 to 197. Subjects' refraction ranged from -6D to -12D. Three studies used EVO Visian ICL (Visian ICL V4c) and two studies used Visian
ICL without knowing the model. The median follow-up duration of five studies ranged from three months to 60 months.

\section{Efficacy and safety index}

All five studies evaluated the efficacy index (Figure 2). Overall, ICL implantation showed a statistically better efficacy index $(\mathrm{MD}=0.09,95 \% \mathrm{CI}: 0.01$ to 0.16$)$ and a better safety index $(\mathrm{MD}=0.08,95 \% \mathrm{CI}: 0.00,0.16)$ than SMILE. Random-effects model were used for 
Table 1 Characteristics of included studies

\begin{tabular}{|c|c|c|c|c|c|c|c|c|c|}
\hline \multirow[t]{2}{*}{ First author } & \multirow[t]{2}{*}{ Publish Year } & \multicolumn{2}{|c|}{ Age (years) } & \multirow[t]{2}{*}{$\mathrm{N}$ (eyes) } & \multirow[t]{2}{*}{ Study design } & \multirow{2}{*}{$\begin{array}{l}\text { Follow-up } \\
\text { time (months) }\end{array}$} & \multicolumn{2}{|l|}{ Myopia range } & \multirow[t]{2}{*}{ ICL type } \\
\hline & & ICL & SMILE & & & & ICL & SMILE & \\
\hline Qin Q & 2019 & 20 to 34 & 20 to 31 & 96 & Observational study & Median: 3 & $-6.25 \mathrm{D}$ to $-10 \mathrm{D}$ & $-6.25 \mathrm{D}$ to $-10 \mathrm{D}$ & Visian ICL(V4C) \\
\hline Niu LL & 2020 & $27.3 \pm 5.5$ & $28.4 \pm 4.2$ & 76 & Observational study & Median: 12 & $-6 D$ to $-9 D$ & $-6 D$ to $-9 D$ & Visian ICL(V4C) \\
\hline Siedlecki J & 2020 & $33.9 \pm 6.4$ & $32.2 \pm 7.6$ & 80 & Observational study & $\begin{array}{l}\text { ICL: } 27.8 \pm 14.3 \\
\text { SMILE: } 26.6 \pm \\
17.7\end{array}$ & $-6 \mathrm{D}$ to $-10 \mathrm{D}$ & $-6 \mathrm{D}$ to $-10 \mathrm{D}$ & Visian ICL \\
\hline Wei R & 2020 & $27.0 \pm 5.3$ & $28.7 \pm 5.0$ & 197 & Observational study & Median: 6 & $-6 D$ to $-10 D$ & $-6 \mathrm{D}$ to $-10 \mathrm{D}$ & Visian ICL(V4c) \\
\hline Jiang Z & 2021 & $26.8 \pm 5.2$ & $28.3 \pm 5.3$ & 106 & $\begin{array}{l}\text { Retrospective case } \\
\text { series }\end{array}$ & Median: 12 & $-6 \mathrm{D}$ to $-12 \mathrm{D}$ & $-6 \mathrm{D}$ to $-12 \mathrm{D}$ & Visian ICL \\
\hline
\end{tabular}

ICL: implantable collamer lens. SMILE: small incision lenticule extraction

\section{Efficacy index}

\begin{tabular}{|c|c|c|c|c|c|c|}
\hline Study & Total & Mean & $\begin{array}{r}\text { ICL } \\
\text { SD }\end{array}$ & Total & Mean & $\begin{array}{r}\text { SMILE } \\
\text { SD }\end{array}$ \\
\hline Qin Q 2019 & 48 & 1.01 & 0.0100 & 48 & 1.00 & 0.0100 \\
\hline Niu LL 2020 & 39 & 1.06 & 0.1500 & 37 & 1.05 & 0.1400 \\
\hline Siedlecki J 2020 & 40 & 1.28 & 0.2400 & 40 & 1.05 & 0.2500 \\
\hline Wei R 2020 & 94 & 1.17 & 0.1600 & 103 & 1.09 & 0.2000 \\
\hline Jiang Z 2021 & 48 & 1.28 & 0.2200 & 58 & 1.13 & 0.2600 \\
\hline $\begin{array}{l}\text { Common effect model } \\
\text { Random effects model } \\
\text { Heterogeneity: } I^{2}=88 \%, \tau\end{array}$ & 269 & & & 286 & & \\
\hline
\end{tabular}

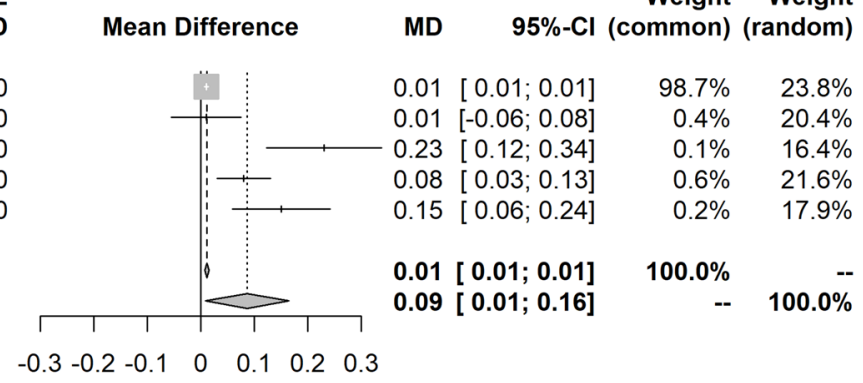

\section{Safety index}

\section{Study}

Qin Q 2019

Niu LL 2020

Siedlecki J 2020

Wei R 2020

Jiang Z 2021

Common effect model 269

Random effects model

Heterogeneity: $I^{2}=88 \%, \tau^{2}=0.0074, p<0.01$

ICL SMILE

Total Mean

$\begin{array}{lllrll}48 & 1.01 & 0.0200 & 48 & 1.00 & 0.0100 \\ 39 & 1.11 & 0.1500 & 37 & 1.13 & 0.1300 \\ 40 & 1.31 & 0.2200 & 40 & 1.10 & 0.2500 \\ 94 & 1.21 & 0.1800 & 103 & 1.12 & 0.1600 \\ 48 & 1.33 & 0.2700 & 58 & 1.17 & 0.2400\end{array}$

286

Fig. 2 Forest plot of comparison on efficacy index and safety index

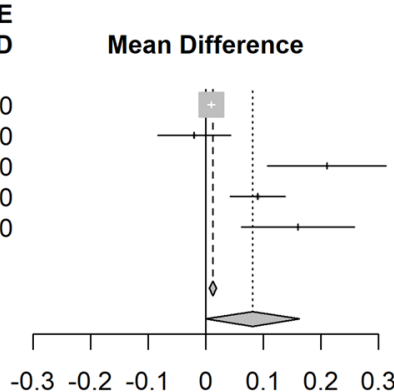

$\begin{array}{rrrr}\text { MD } & \text { 95\%-Cl } \begin{array}{r}\text { Weight } \\ \text { (common) }\end{array} & \begin{array}{c}\text { Weight } \\ \text { (random) }\end{array} \\ 0.01[0.00 ; 0.02] & 96.6 \% & 23.3 \% \\ -0.02[-0.08 ; 0.04] & 1.0 \% & 20.5 \% \\ 0.21[0.11 ; 0.31] & 0.4 \% & 17.0 \% \\ 0.09[0.04 ; 0.14] & 1.7 \% & 21.6 \% \\ 0.16[0.06 ; 0.26] & 0.4 \% & 17.5 \% \\ & & & \\ \mathbf{0 . 0 1}[0.01 ; 0.02] & 100.0 \% & -- \\ \mathbf{0 . 0 8}[\mathbf{0 . 0 0} \mathbf{0 . 1 6}] & -- & \mathbf{1 0 0 . 0 \%}\end{array}$

$\begin{array}{lllllll}-0.3 & -0.2 & -0.1 & 0 & 0.1 & 0.2 & 0.3\end{array}$

meta-analysis due to a large heterogeneity across studies $\left(\mathrm{I}^{2}>50 \%\right)$.

\section{CDVA}

Three studies $[16,18,20]$ assessed change in Snellen lines of CDVA (Fig. 3), the heterogeneity across three studies was small $\left(\mathrm{I}^{2}=50 \%\right)$, so a fixed-effects model was applied. Compared with SMILE-treated eyes, more ICL-treated eyes gained one or more lines of CDVA $(R R=1.54$, 95\%CI:1.28 to 1.86), more ICL-treated eyes gained two or more lines of CDVA $(\mathrm{RR}=2.09,95 \% \mathrm{CI}: 1.40$ to 3.13$)$, less ICL-treated eyes lost one or more lines of CDVA $(\mathrm{RR}=0.17,95 \% \mathrm{CI}: 0.05$ to 0.63$)$. No eyes lost two or more Snellen lines in each group.

\section{Predictability}

Four studies (except for study of Qin Q [21]) reported predictability of both treatments, difference of postoperative SER and attempted SER was used to evaluate the predictability. The $\mathrm{I}^{2}$ of predictability of within \pm $0.5 \mathrm{D}$ and within $\pm 1 \mathrm{D}$ were $80 \%$ and $0 \%$ respectively, thus a random-effects model and a fixed-effects model were 


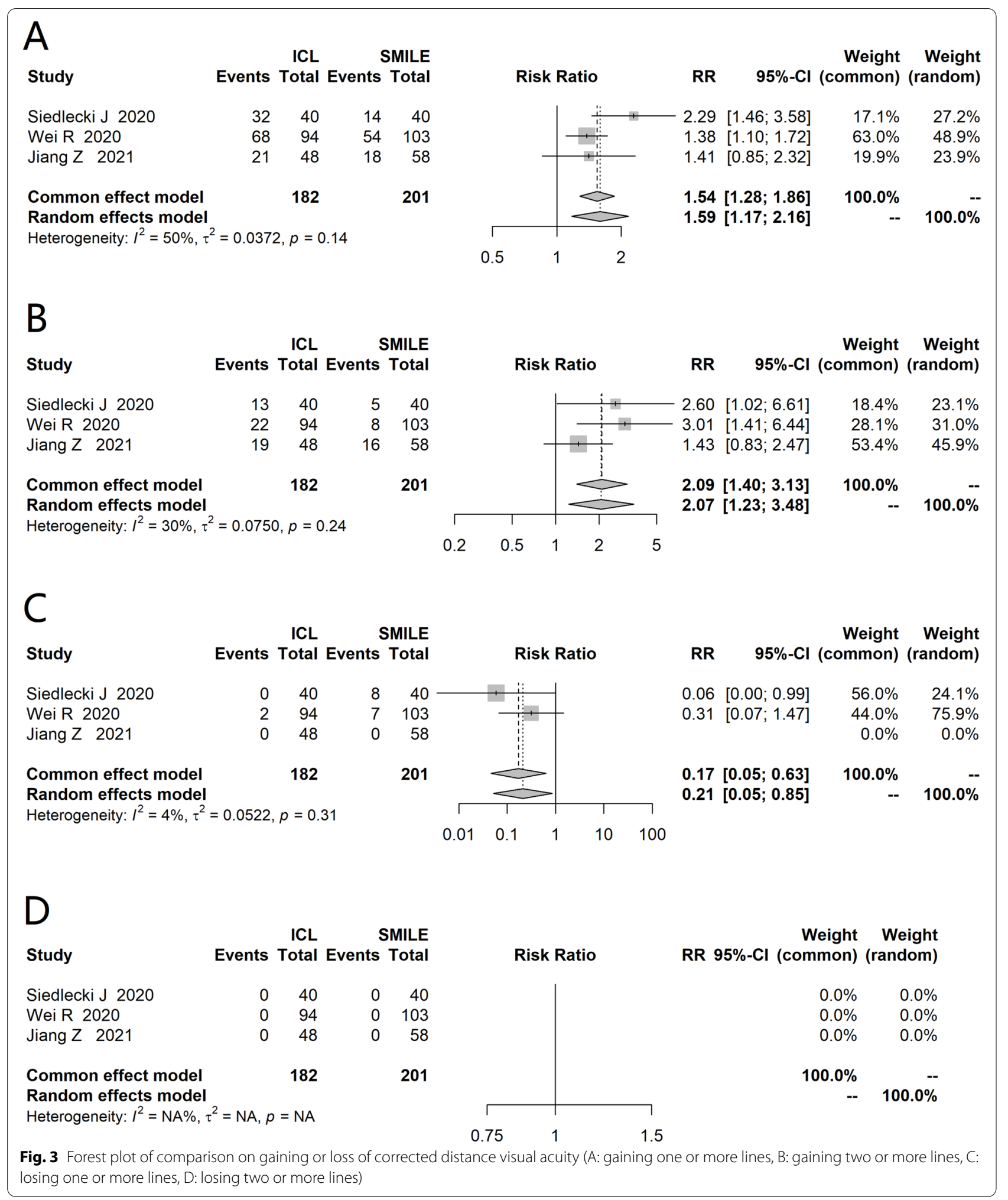

used to conduct meta-analysis respectively. Forest plot (Fig. 4) showed no statistical difference in predictability of within $\pm 0.5 \mathrm{D}$ between two treatments $(R R=1.13$,
95\%CI: 0.94 to 1.36$)$, there was no statistical difference in predictability of within $\pm 1 \mathrm{D}$ either $(\mathrm{RR}=1.00,95 \% \mathrm{CI}$ : 0.98 to 1.02$)$. 


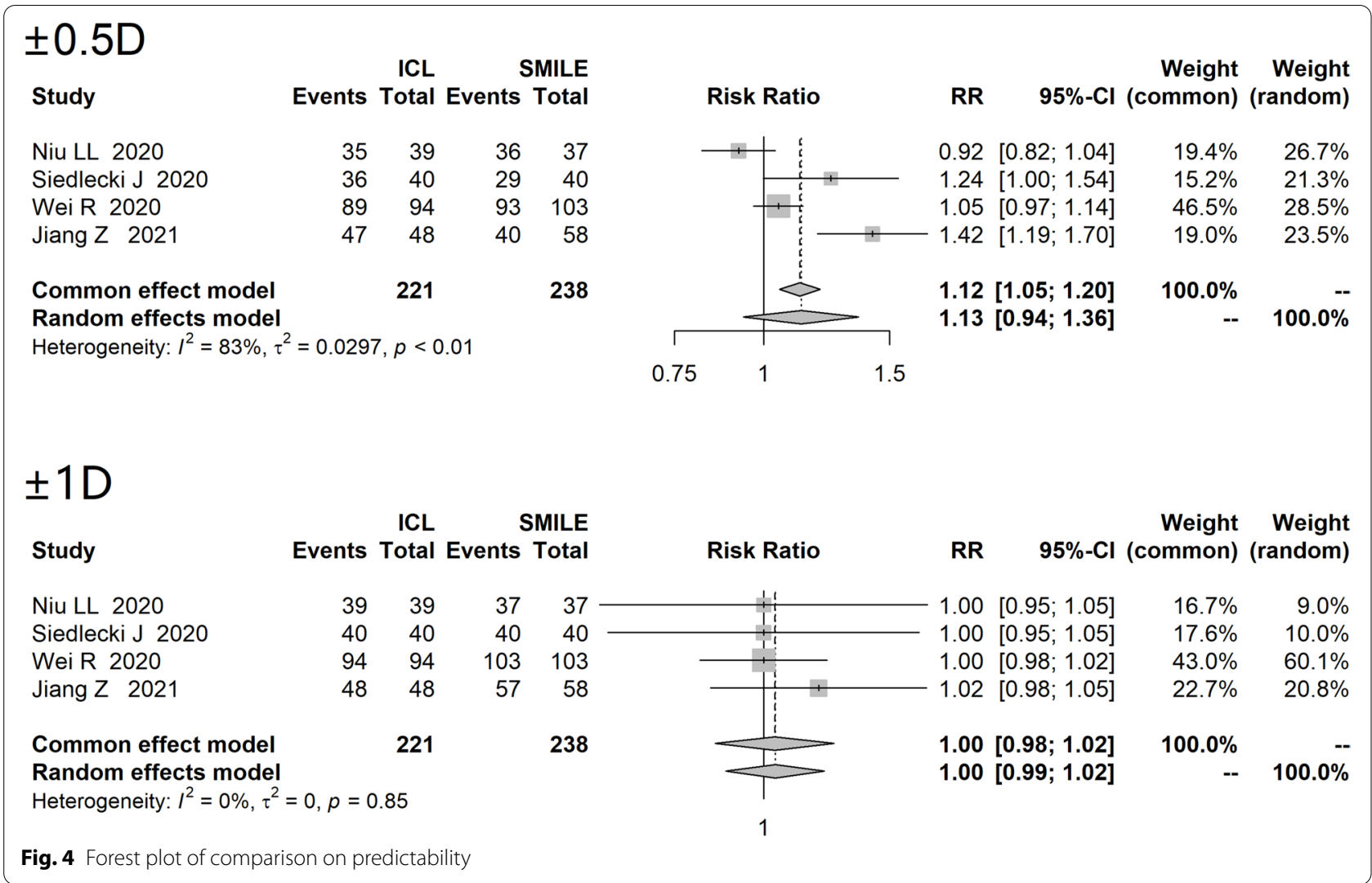

Halos

Three studies $[13,16,18]$ evaluated halos after treatment, ICL implantation showed statistically higher risk of halos than SMILE (Fig. 5), the RR was 1.79 (95\%CI: 1.48 to $2.16)$, there was no heterogeneity across included studies $\left(\mathrm{I}^{2}=0 \%\right)$.

\section{Increase of total HOAs}

Two studies assessed change of total HOAs, the heterogeneity across two studies was large $\left(\mathrm{I}^{2}=97 \%\right)$, thus a random-effects model was applied. ICL-treated eyes showed a smaller increase of total HOAs (Fig. 6) than SMILEtreated eyes $(\mathrm{MD}=-0.23,95 \% \mathrm{CI}:-0.42,-0.03)$.

\section{Other outcomes}

For other outcomes including cornea endothelial cell loss, complications including cataract and dry eye, visual quality including objective scatter index (OSI) and modulation transfer function cut-off frequency $\left(\mathrm{MTF}_{\text {cut-off }}\right)$ value, no pooled MD or pooled RR was calculated due to limited data. Qin Q and coworkers reported no significant endothelial cell density loss in either ICL-treated

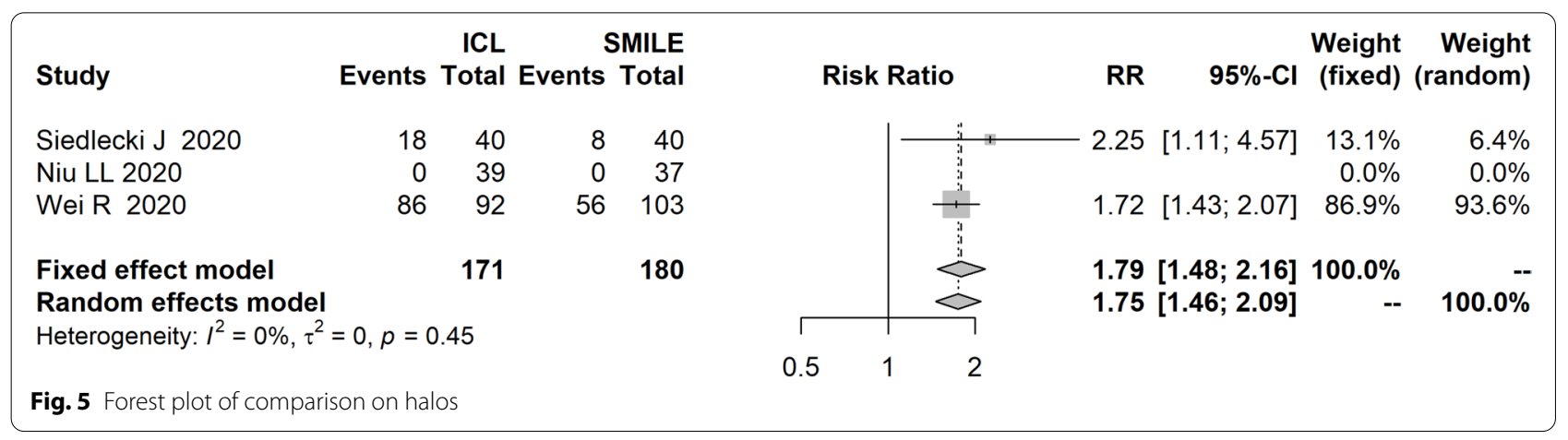




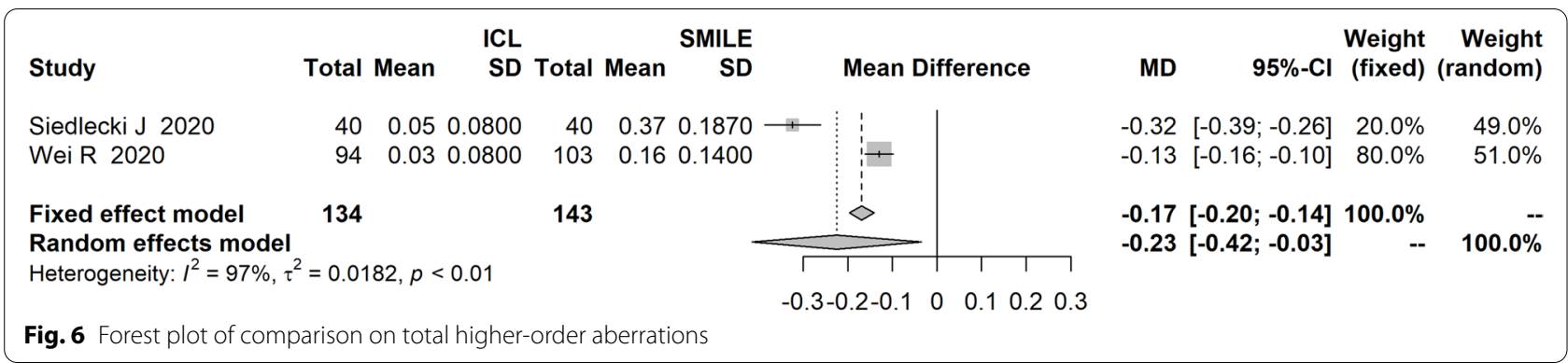

eyes or SMILE-treated eyes. Niu LL [13] and coworkers reported no statistical difference between ICL implantation and SMILE in change of either OSI or MTF cut-off $_{\text {- }}$ before and after surgery. None study reported occurrence of cataract or dry eye in each treatment group.

\section{Publication bias test}

Publication bias was checked using Egger's test, no publication bias was found by Egger's test ( $p>0.05$ ).

\section{Discussion}

High myopia, predisposed by genetic and environmental factors [22-24], poses challenge to clinical treatment, clinicians have to balance the benefit and risk since no choice is perfect. In this meta-analysis, we compared two popular options (SMILE and ICL implantation) for high myopia correction to help doctors make better decision. Efficacy index, calculated from visual acuity, is the most important outcome reflecting the efficacy of treatments. By this review, ICL implantation showed a better efficacy index than SMILE, which indicated that ICL implantation might be more efficient than SMILE for high myopia correction. ICL implantation also showed a better performance on safety index than SMILE. Besides, ICL implantation tended to come with a lower risk of visual acuity loss than SMILE, only 1.09\% (2/182) of ICL-treated eyes lost one or more lines of CDVA, while the percentage reached 7.46\% (15/201) in SMILE-treated eyes.

The predictability on SER reflects the accuracy of treatments, both ICL implantation and SMILE should achieve good accuracy according to their design principles. In this meta-analysis, both treatments showed good and equal performance on predictability, 93.67\% (207/221) ICL-treated eyes and 83.19\% (198/238) SMILE-treated eyes achieved a predictability of within $\pm 0.5 \mathrm{D}, 100 \%$ $(221 / 221)$ and $99.58 \%(237 / 238)$ achieved a predictability of within $\pm 1 \mathrm{D}$. Many previous studies reported similar findings, usually more than 95\% ICL-treated eyes were within $\pm 1.00 \mathrm{D}$ of the intended refraction [11, 25-27]. A large retrospective study included 722 SMILE-treated high myopic eyes, $98 \%$ were within $\pm 1.00 \mathrm{D}$ of the intended refraction [28].
This meta-analysis showed ICL implantation leads to a smaller change in total HOAs than SMILE, this is reasonable since theoretically ICL implantation does not cause damage to the physiological structure of the cornea itself, thus eyeballs are able to preserve the adjustment ability, ensuring that subjects could obtain a more ideal visual quality. On the contrary, SMILE causes damage to the corneal surface morphology, and thus leads to change of HOAs. Evidence from previous studies supported the theory, this meta-analysis showed a smaller increase in total HOAs in ICL-treated eyes compared with SMILE-treated eyes, many other studies reported similar findings, usually, there was no significant increase of HOAs ICL-treated eyes [29, 30], while for SMILE-treated high myopic eyes, significant increase of total HOAs were commonly reported [31-34].

Contrast sensitivity, OSI, and MTF cut-off, $_{\text {, }}$ are also important reflections of visual quality, however, no quantitative conclusion was drawn in this meta-analysis because few studies assessed these parameters. None of the included study performed contrast sensitivity test, Igarashi A [35] and coworkers reported that ICL implantation improved the contrast sensitivity while SMILE decreased the contrast sensitivity, Shin JY [36] and coworkers reported that ICL implantation induced fewer ocular and corneal HOAs, which resulted in a better contrast sensitivity at mesopic levels. Current evidence [36] showed no difference on OSI between ICL implantation and SMILE. But in terms of $\mathrm{MTF}_{\text {cut-off }}$ value, evidence was contradicted, Qin Q [21] and coworkers reported that the postoperative $\mathrm{MTF}_{\text {cut-off }}$ value of ICL-treated eyes was higher than SMILE-treated eyes, while Niu LL [13] and coworker found no significant difference between two treatments. However, given that either study of Qin Q [21] or study of Niu LL [13] was observational studies, which meaning the preoperative $\mathrm{MTF}_{\text {cut-off }}$ value of two treatments might not be balanced, thus assessing the change in $\mathrm{MTF}_{\text {cut-off }}$ value and change in OSI might be more meaningful, Niu LL [13] and coworkers reported no significant change before and after surgery, either for $\mathrm{MTF}_{\text {cut-off }}$ value or OSI. 
Complications are also important reflections of treatments' safety, endothelial cell density loss was thought to be a main backward of ICL implantation [37]. In this meta-analysis, Qin Q's study [21] reported no significant decrease before and after surgery either in the ICL group or SMILE group, the current evidence is not enough to assess the safety of both treatments. Halos were reported to be the leading complication of ICL implantation, the prevalence rates ranged from $15.2 \%[38,39]$ to $93.5 \%[16$, $18]$. By this meta-analysis, up to $60.81 \%(104 / 171)$ ICLtreated eyes and $35.56 \%(64 / 180)$ SMILE-treated eyes perceived halos, in study of Wei R [18], the halos rates in ICL group and SMILE group were even as high as $93.5 \%$ and $54.4 \%$, the reason might that the follow-up time was short (six months), as halos were commonly seen in the early period after ICL and SMILE surgery. Although no cataract formation was reported in either ICL-treated eyes or SMILE-treated eyes in five studies, it doesn't represent that cataract is not a concern since the follow-up duration was usually one year or less except for study of Siedlecki J [16] (about two-year follow-up), Guber I $\mathrm{I}^{39}$ and coworkers reported the lens opacity rate could reach $40.9 \%$ in 133 high myopic eyes 10 years after ICL implantation, 18 eyes of which underwent phacoemulsification.

In conclusion, both ICL implantation and SMILE had satisfying and equal performance on refraction control. ICL implantation came with a higher risk of halos, but was better than SMILE on efficacy, safety, CDVA improvement and total HOAs control. Overall, for high myopia correction, ICL implantation might be a better choice than SMILE. However, the conclusion came from observational studies with relatively short-term followup, evidence from randomized controlled trials and longterm studies is still needed.

\section{Search strategy}

\section{PubMed, Science direct, Embase}

((posterior chamber phakic intraocular lens) OR piol OR (implantable collamer lens) OR ICL OR ticl OR V4 OR V4C OR STAAR) AND ((small-incision lenticule extraction) OR smile OR (cornea refractive surgery)) AND (myopia OR (refractive errors) OR refraction)

\section{Cochrane Central Register of Controlled Trials}

\#1posterior chamber phakic intraocular lens \#2piol

\#3implantable collamer lens

\#4ICL

\#5ticl

\#6V4
\#7V4C

\#8STAAR

$\# 9 \# 1$ or $\# 2$ or \#3 or \#4 or \#5 or \#6 or \#7 or \#8

\#10small-incision lenticule extraction

$\# 11$ smile

$\# 12$ cornea refractive surgery

$\# 13 \# 10$ or \#11 or \#12

\#14myopia

\#15refractive errors

\#16refraction

$\# 17$ \#14 or \#15 or \#16

\#31\#9 AND \#13 AND \#17

\section{Acknowledgements \\ None}

Authors' contributions

Concept (Xiu Hua Wan, Ningli Wang, Zi-Bing Jin), literature search (Kai Cao, Shanshan Jin), data extraction (Kai Cao, Shanshan Jin), data analysis (Kai Cao), manuscript writing (Kai Cao, Xiu Hua Wan, Ningli Wang, Zi-Bing Jin, Jingshang Zhang, Jinda Wang, Shuying Chen), English polishing (Mayinuer Yusufu).

Funding

None

Availability of data and materials

Data would be available upon reasonable request

\section{Declarations}

Ethics approval and consent to participate

Not applicable

\section{Competing interests}

There is no conflict of interest.

Received: 31 August 2021 Accepted: 6 December 2021

Published online: 27 December 2021

References

1. Dishler JG, Slade S, Seifert S, Schallhorn SC. Small-Incision Lenticule Extraction (SMILE) for the Correction of Myopia with Astigmatism: Outcomes of the United States Food and Drug Administration Premarket Approval Clinical Trial. Ophthalmology. 2020;127(8):1020-34.

2. Randleman JB. Small Incision Lenticule Extraction (SMILE): What Now? What Next? Ophthalmology. 2020;127(8):1035-6.

3. Yin Y, Lu Y, Xiang A, et al. Comparison of the optical quality after SMILE and FS-LASIK for high myopia by OQAS and iTrace analyzer: a one-year retrospective study. Bmc Ophthalmol. 2021;21(1):292.

4. Qian Y, Chen X, Naidu RK, Zhou X. Comparison of efficacy and visual outcomes after SMILE and FS-LASIK for the correction of high myopia with the sum of myopia and astigmatism from -10.00 to -14.00 dioptres. Acta Ophthalmol. 2020;98(2):e161-72.

5. Denoyer $A$, Landman $E$, Trinh $L$, et al. Dry eye disease after refractive surgery: comparative outcomes of small incision lenticule extraction versus LASIK. Ophthalmology. 2015;122(4):669-76.

6. Wang S, Dai J, Chu R, Zhou X. Long-term outcome of epi-LASIK for high myopia. Eur J Ophthalmol. 2012;22(Suppl 7):S98-105.

7. Rosman M, Alio JL, Ortiz D, Perez-Santonja JJ. Comparison of LASIK and photorefractive keratectomy for myopia from -10.00 to -18.00 diopters 10 years after surgery. J Refract Surg. 2010;26(3):168-76.

8. Randleman JB, Russell B, Ward MA, et al. Risk factors and prognosis for corneal ectasia after LASIK. Ophthalmology. 2003;1 10(2):267-75. 
9. Sanders DR, Brown DC, Martin RG, et al. Implantable contact lens for moderate to high myopia: phase 1 FDA clinical study with 6 month follow-up. J Cataract Refract Surg. 1998;24(5):607-11.

10. Kocova H, Vlkova E, Michalcova L, Motyka O. Implantation of posterior chamber phakic intraocular lens for myopia and hyperopia - long-term clinical outcomes. J Fr Ophtalmol. 2017;40(3):215-23.

11. Yan Z, Miao H, Zhao F, et al. Two-Year Outcomes of Visian Implantable Collamer Lens with a Central Hole for Correcting High Myopia. J Ophthalmol. 2018:2018:8678352.

12. Kamiya K, Shimizu K, Igarashi A, Komatsu M. Comparison of Collamer toric implantable [corrected] contact lens implantation and wavefrontguided laser in situ keratomileusis for high myopic astigmatism. J Cataract Refract Surg. 2008:34(10):1687-93.

13. Niu L, Miao H, Tian M, et al. One-year visual outcomes and optical quality of femtosecond laser small incision lenticule extraction and Visian Implantable Collamer Lens (ICL V4c) implantation for high myopia. Acta Ophthalmol. 2020.

14. Bhikoo R, Rayner S, Gray T. Toric implantable collamer lens for patients with moderate to severe myopic astigmatism: 12-month follow-up. Clin Exp Ophthalmol. 2010;38(5):467-74.

15. Abdelmassih Y, El-Khoury S, Chelala E, et al. Toric ICL Implantation After Sequential Intracorneal Ring Segments Implantation and Corneal Crosslinking in Keratoconus: 2-Year Follow-up. J Refract Surg. 2017;33(9):610-6.

16. Siedlecki J, Schmelter V, Mayer WJ, et al. SMILE Versus Implantable Collamer Lens Implantation for High Myopia: A Matched Comparative Study. J Refract Surg. 2020;36(3):150-9.

17. Moshirfar M, Somani AN, Motlagh MN, et al. Comparison of FDAReported Visual and Refractive Outcomes of the Toric ICL Lens, SMILE, and Topography-Guided LASIK for the Correction of Myopia and Myopic Astigmatism. J Refract Surg. 2019;35(11):699-706.

18. Wei R, Li M, Zhang H, et al. Comparison of objective and subjective visual quality early after implantable collamer lens V4C (ICL V4c) and small incision lenticule extraction (SMILE) for high myopia correction. Acta Ophthalmol. 2020.

19. Cao K, Friedman DS, Jin S, et al. Multifocal versus monofocal intraocular lenses for age-related cataract patients: a system review and metaanalysis based on randomized controlled trials. Surv Ophthalmol. 2019;64(5):647-58.

20. Jiang Z, Wang H, Luo DQ, Chen J. Optical and visual quality comparison of implantable collamer lens and femtosecond laser assisted laser in situ keratomileusis for high myopia correction. Int J Ophthalmol. 2021;14(5):737-43.

21. Qin $Q$, Bao L, Yang L, et al. Comparison of visual quality after EVO-ICL implantation and SMILE to select the appropriate surgical method for high myopia. Bmc Ophthalmol. 2019;19(1):21.

22. Cai XB, Zheng YH, Chen DF, et al. Expanding the Phenotypic and Genotypic Landscape of Nonsyndromic High Myopia: A Cross-Sectional Study in 731 Chinese Patients. Invest Ophthalmol Vis Sci. 2019;60(12):4052-62.

23. Cai XB, Shen SR, Chen DF, et al. An overview of myopia genetics. Exp Eye Res. 2019;188:107778.

24. Jin ZB, Wu J, Huang XF, et al. Trio-based exome sequencing arrests de novo mutations in early-onset high myopia. Proc Natl Acad Sci U S A. 2017;114(16):4219-24

25. Li K, Wang Z, Zhang D, et al. Visual Outcomes and Corneal Biomechanics after Visian V4c Phakic Intraocular Lens Implantation in subclinical keratoconus. J Cataract Refract Surg. 2020.

26. Kamiya K, Shimizu K, Kobashi H, et al. Clinical outcomes of posterior chamber toric phakic intraocular lens implantation for the correction of high myopic astigmatism in eyes with keratoconus: 6-month follow-up. Graefes Arch Clin Exp Ophthalmol. 2011:249(7):1073-80.

27. Chaitanya SR, Anitha V, Ravindran M, et al. Safety and efficacy of toric implantable collamer lens $\mathrm{V} 4 \mathrm{c}$ model - A retrospective South Indian study. Indian J Ophthalmol. 2020;68(12):3006-11.

28. Hansen RS, Lyhne N, Grauslund J, Vestergaard AH. Small-incision lenticule extraction (SMILE): outcomes of 722 eyes treated for myopia and myopic astigmatism. Graefes Arch Clin Exp Ophthalmol. 2016;254(2):399-405.

29. Wan T, Yin H, Wu Z, Yang Y. Comparative Study of Implantable Collamer Lens Implantation in Treating Four Degrees of Myopia: Six-Month Observation of Visual Results, Higher-Order Aberrations, and Amplitude of Accommodation. Curr Eye Res. 2019:1-8.
30. Hashemian SJ, Farrokhi H, Foroutan A, et al. Ocular higher-order aberrations changes after implantable collamer lens implantation for high myopic astigmatism. J Curr Ophthalmol. 2018;30(2):136-41.

31. Lee $H$, Yong KD, Reinstein DZ, et al. Comparing corneal higher-order aberrations in corneal wavefront-guided transepithelial photorefractive keratectomy versus small-incision lenticule extraction. J Cataract Refract Surg. 2018;44(6):725-33.

32. Qin B, Li M, Chen X, et al. Early visual outcomes and optical quality after femtosecond laser small-incision lenticule extraction for myopia and myopic astigmatism correction of over -10 dioptres. Acta Ophthalmol. 2018;96(3):e341-6.

33. Miraftab M, Hashemi H, Aghamirsalim M, et al. Matched comparison of corneal higher order aberrations induced by SMILE to femtosecond assisted LASIK and to PRK in correcting moderate and high myopia: $3.00 \mathrm{~mm}$ vs. $6.00 \mathrm{~mm}$. Bmc Ophthalmol 2021;21(1):216.

34. Xia F, Qin B, Shang J, et al. Four-Year Outcomes of Small Incision Lenticule Extraction for Extreme High Myopia and Myopic Astigmatism. Front Med (Lausanne). 2020;7:575779.

35. Igarashi A, Kamiya K, Shimizu K, Komatsu M. Visual performance after implantable collamer lens implantation and wavefront-guided laser in situ keratomileusis for high myopia. Am J Ophthalmol. 2009;148(1):164-70

36. Shin JY, Ahn H, Seo KY, et al. Comparison of higher order aberrations after implantable Collamer Lens implantation and wavefront-guided LASEK in high myopia. J Refract Surg. 2012;28(2):106-11.

37. Fuller DJ, Carper SW, Clay L, et al. Polyamine regulation of heatshock-induced spermidine N1-acetyltransferase activity. Biochem J. 1990;267(3):601-5.

38. Bamashmus MA, Al-Arabi AH, Alawad MA. Visual outcomes and patient satisfaction after implantable collamer lens and Toric implantable collamer lens correction for moderate to high myopia and myopic astigmatism. Saudi Med J. 2013;34(9):913-9.

39. Guber I, Mouvet V, Bergin C, et al. Clinical Outcomes and Cataract Formation Rates in Eyes 10 Years After Posterior Phakic Lens Implantation for Myopia. Jama Ophthalmol. 2016;134(5):487-94.

\section{Publisher's Note}

Springer Nature remains neutral with regard to jurisdictional claims in published maps and institutional affiliations.

Ready to submit your research? Choose BMC and benefit from

- fast, convenient online submission

- thorough peer review by experienced researchers in your field

- rapid publication on acceptance

- support for research data, including large and complex data types

- gold Open Access which fosters wider collaboration and increased citations

- maximum visibility for your research: over $100 \mathrm{M}$ website views per year

At BMC, research is always in progress.

Learn more biomedcentral.com/submissions 